\title{
Pose Estimation of Eyes for Particle Beam Treatment of Tumors
}

\author{
B. P. Selby ${ }^{1}$, G. Sakas ${ }^{1}$, S. Walter ${ }^{1}$, W.-D. Groch ${ }^{2}$, U. Stilla ${ }^{3}$ \\ ${ }^{1}$ Cognitive Computing and Medical Imaging, Fraunhofer IGD, Darmstadt, Germany \\ ${ }^{2}$ Fachbereich Informatik, University of Applied Sciences, Darmstadt, Germany \\ ${ }^{3}$ Photogrammetry and Remote Sensing, Technische Universität Muenchen, Germany \\ Email: pselby@medcom-online.de
}

\begin{abstract}
To assure a correct position and orientation of the patient's eye in radiation treatment, a new approach in image-guided radiotherapy is used to determine the misalignment of the eye and the respective correction for the patient support devices. New methods allow correcting the misalignment of the patient's eye full-automatically. Therefore, metallic clips attached to the patient's eyeball are detected in a CT and in two digital X-ray images. Corresponding pairs of clip positions found in the $\mathrm{X}$-ray images are then transferred into the $3 \mathrm{D}$ space of the $\mathrm{CT}$ volume via inverse projection and compared to the clips found in the CT slices. If several combinations of correspondences are possible, we use a temporary pre-registration to assure that the correct back-projections will be obtained. A rigid, point-set based 6 degrees of freedom registration of the back-projected clip positions with the CT clips allows determining the misalignment of the eye tumor which consists of three shifts and three rotations. These transformations are transformed into parameters for a treatment chair and an eye fixation designated to control placement and orientation of the eye during treatment.
\end{abstract}

\section{Introduction}

Modern particle beam based treatment techniques allow an accurate application of the treatment dose onto carcinogen tissue. A tumor can be treated by a proton beam line by line with a geometric accuracy much better than $1.0 \mathrm{~mm}$. This is advantageous for radio-oncological treatment of organs where sensible tissue near the treatment target has to be saved from being irradiated, as for example the optic nerve in the treatment of eye tumors.

This accuracy is limited by treatment position set-up errors originating from aberrations of the location of the planned radiation target relative to the particle beam. Strategies like tracking of external markers or fixation of the patient's body do not suffice the requirement of high set-up precision and are not applicable in the case of eye tumor treatment. Besides that, the eye orientation may change according to patient movements, during a time consuming manual set-up procedure.

To overcome these problems, a new approach exploits the high-resolution slices of a planning CT for eye treatment and two X-ray images acquired from 
within the treatment device. About 5 clips of tantalum or titanium, attached to the treated eye are detected in the $\mathrm{CT}$ and the X-ray images acquired shortly before the treatment takes place or during the treatment session, to verify the correctness of the eye alignment.

Based on an automatic registration of the clip positions, an alignment correction for the eye is calculated in 6 degrees of freedom, 3 shifts and 3 rotations, with a very high precision and reliability.

\section{State of the art new contribution}

The correctness of the position of a patient's eye undergoing particle beam treatment relies on the fixation equipment and the X-ray images that are compared to a CT image series in order to detect remaining misalignments. While the fixation equipment assures an initial set-up preciseness of about $0.5 \mathrm{~mm}$ [1], a fine-tuning is done by manual step-by-step correction, reducing the remaining misalignment between CT and X-ray images [2]. This procedure can be time consuming and therefore limits the throughput of patients in a commercial health center and it leads to a high number of X-ray acquisitions and the respective application of X-ray doses on the patient. Set-up errors from the manual correction cannot be eliminated, as the correctness of the alignment depends on the operator's judgment.

Automatic position correction procedures [3, 4] based on mutual information registration are not applicable in the scope of eye treatment, as they strongly rely on bony structures instead on the relatively homogenous tissue of the eyeball.

The automatic detection and registration of eye clips proposed herewith allows a very fast and accurate position and orientation correction for the eye in 6 degrees of freedom.

\section{Methods}

The automatic procedure consists of the following working steps: Segmentation of clips in a CT volume; Segmentation of clips in two X-ray images; Back projection of the clip positions from the X-rays; Registration of the resulting point-sets; Translation of the registration result into appropriate set-up correction parameters.

\subsection{Clip segmentation in CT series}

To enable a very fast detection, clips are segmented in the CT data using different levels of the volume resolution. The $\mathrm{CT}$ is resampled to a $4 \mathrm{D}$ pyramid containing several instances of the volume, each with a different resolution. The search for a clip starts at a low-resolution level. As soon as a potential clip voxel is found, the search continues at a higher resolution. To determine if a voxel belongs to a clip, two Hounsfield thresholds are used. A voxel between those thresholds is 
Fig. 1. Results of segmentations with different thresholds (left); Patient chair (right)
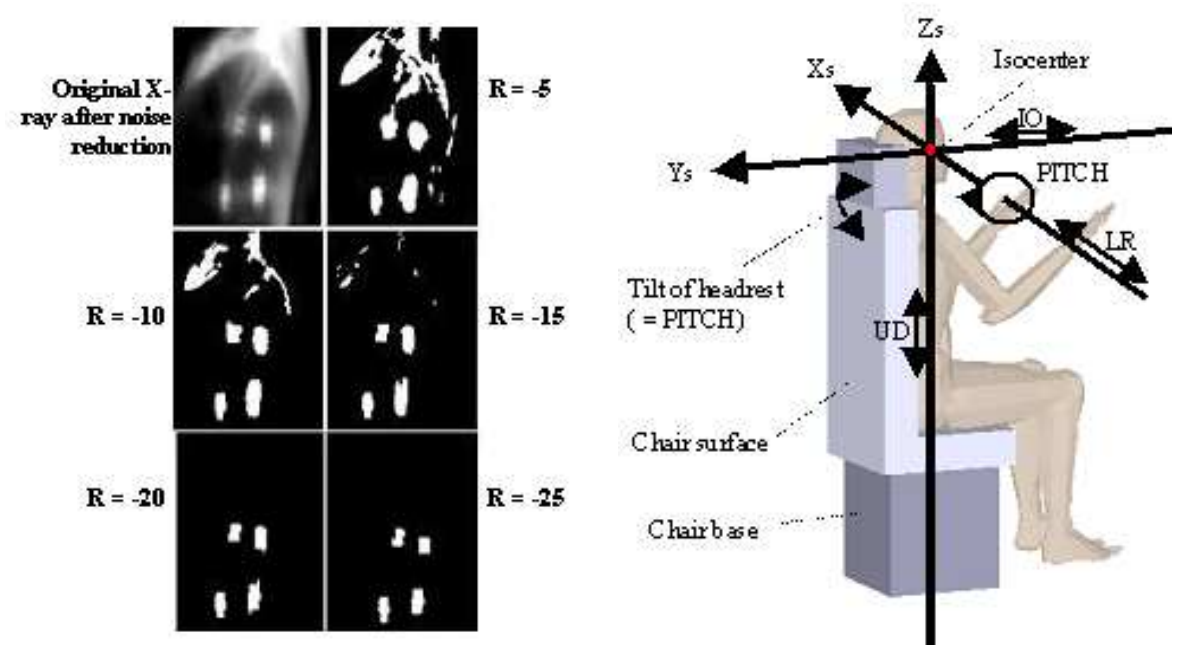

considered to belong to a clip. As soon as one clip is found the thresholds are adapted, assuming, that upcoming clips are of the same material and will be represented by similar Hounsfield values. If no clip is found, the thresholds are modified and the search continues.

\subsection{Clip segmentation in X-ray images}

For detecting the clips in the X-ray images, two different approaches are combined. Using a Harris Corner Detector [5] potential clip corners are identified. Then several segmentations are performed. In each segmentation a different gray value threshold is used to identify potential clip pixels. In addition the distance of a potential clip pixel to its next non-clip border is evaluated. Again different thresholds $R$ are used for the accepted distance of a potential clip pixel to its border pixel (Fig. 1, left).

The information about area and periphery of all segmentations for one clip is combined and the results most likely representing a clip are maintained.

\subsection{Back projection}

To compare the clips detected in the X-ray images with the clips from the CT, an inverse projection is performed for corresponding pairs of both X-rays.

Because it is not always clear, which clips correspond to each other, it is possible that one clip becomes a member in several back projection results. This is the case, if several clips are projected onto a horizontal line in the plane of one flat panel. Performing a separate registration for each possible result set of the inverse projection solves this problem. Only the registration that tends to result in an acceptable patient set-up is used for the final set-up correction. 


\subsection{Registration}

Two sets of points in the 3D space of the CT are used to calculate 3 shifts and 3 rotations, which map one point-set onto the other as good as possible. The remaining mapping error serves as an indicator for the quality of the patient setup calculation. We use a Downhill Simplex optimization [6] to minimize an error for the misalignment between back projected point-set A and the original CT clip positions B. The error metric bases on the undirected Hausdorff Distance $[7]$

$$
H(A, B)=H(B, A)=\max (h(A, B), h(B, A))
$$

$$
\text { with } h(A, B)=\max _{a \in A} \min _{b \in B}(\|a-b\|) \text { and } h(B, A)=\max _{b \in B} \min _{a \in A}(\|b-a\|)
$$

To avoid local minima we use the square sum of the undirected Hausdorff Distance of rank 0 to $\mathrm{k}$, where $\mathrm{k}$ denotes the minimum of the number of clips detected in the X-ray images and the CT slices.

\subsection{Calculation of the correction}

The patient support consists of the patient chair, an adjustable headrest and a fixation light. The patient is advised to focus the fixation light, so it can be used to modify the orientation of the eyeball.

The results from the registration are now used to compute a correction for the set-up parameters of these devices (Fig. 1, right). The possible degrees of freedom are:

- Chair rotation around vertical axis and translation along X-, Y- and Z-axis

- Tilt of the headrest

- Fixation light height and tilt angle to the left or the right

As chair parameter adjustment can be redundant to the eye alignment, it is possible to set preferences for the set-up parameters. A simulated annealing algorithm [6] is used to adjust the chair parameters step by step to model the eye transformation.

A remaining eye torsion, which is a rotation of the eye around the vector in viewing direction, cannot be corrected by patient support adjustment. Together with the remaining error of the registration point-set mapping, a large eye torsion value is used to indicate a bad patient alignment.

\section{Results}

Tests have been performed, using CT datasets $(0.2 \mathrm{~mm}$ slice distance $)$ and $\mathrm{X}$ rays of a pig's eye attached with 4 and 5 tantalum clips (Fig. 2). In all cases it was possible to detect all clips and to perform a correct mapping of the back projected clip positions (Tab. 1). 
Table 1. Correction results for different set-up errors

\begin{tabular}{ccccc}
\hline $\begin{array}{c}\text { Number } \\
\text { of clips }\end{array}$ & $\begin{array}{c}\text { Total (intended) } \\
\text { set-up error } \\
\text { (Rotation /Shift) }\end{array}$ & $\begin{array}{c}\text { Remaining error } \\
\text { after Correction } \\
\text { (Rotation/Shift) }\end{array}$ & $\begin{array}{c}\text { Indicated quality } \\
\text { (0 to 100\%) }\end{array}$ & $\begin{array}{c}\text { Calculation- } \\
\text { time } \\
\text { in seconds }\end{array}$ \\
\hline 4 & $2^{\circ} / 2 \mathrm{~mm}$ & $0.05^{\circ} / 0.20 \mathrm{~mm}$ & $87 \%$ & 1 \\
4 & $5^{\circ} / 10 \mathrm{~mm}$ & $0.09^{\circ} / 0.25 \mathrm{~mm}$ & $90 \%$ & 1 \\
4 & $10^{\circ} / 20 \mathrm{~mm}$ & $0.20^{\circ} / 0.20 \mathrm{~mm}$ & $88 \%$ & 2 \\
5 & $2^{\circ} / 2 \mathrm{~mm}$ & $0.05^{\circ} / 0.21 \mathrm{~mm}$ & $95 \%$ & 2 \\
5 & $5^{\circ} / 10 \mathrm{~mm}$ & $0.05^{\circ} / 0.13 \mathrm{~mm}$ & $91 \%$ & 3 \\
5 & $10^{\circ} / 20 \mathrm{~mm}$ & $0.18^{\circ} / 0.18 \mathrm{~mm}$ & $87 \%$ & 3 \\
\hline
\end{tabular}

\section{Discussion}

It becomes apparent, that the automatic correction is superior to conventional manual correction techniques and allows accomplishing a correction with only one single set of X-ray images, which reduces the $\mathrm{X}$-ray radiation applied to the patient. The proposed methods are suitable for accurate and reliable position

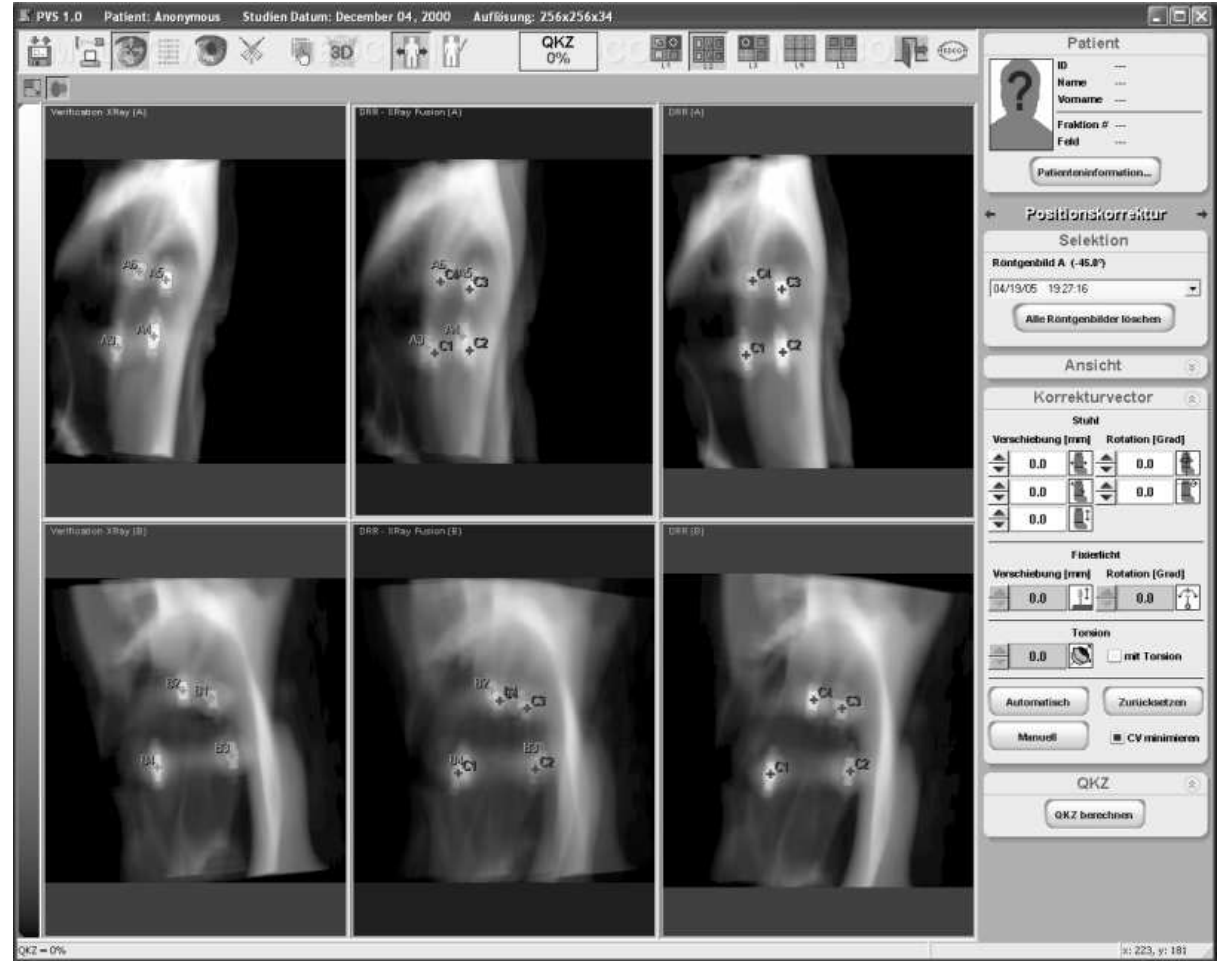

Fig. 2. Images of pig's eye with 4 clips: X-rays (left), fused (center) DRRs (right) 
correction within seconds. The results show, that an increasing number of clips leads to a slightly more accurate correction, but increases the calculation time. Evaluations in real treatment situations still have to be done. Remaining errors result from the slice distance of $0.2 \mathrm{~mm}$. For higher accuracy, usage of CTs with a higher resolution is suggested.

The methods have been tested with $2.5 \mathrm{~mm}$ tantalum clips, which could be detected relatively easy. Further efforts have to be done to assure a correct detection for the wide variety of applicable metal clips. Under adverse circumstances, as when clips are occluded by other clips or by bony structures of the skull, not all clips can be detected, which results, depending on the total number of clips used, in results less accurate.

\section{References}

1. Verhey LJ, Goitein M, McNulty P, Munzenrider JE, Suit HD. Precise positioning of patients for radiation therapy. Int J Radiation Oncology Biol Phys 1982;8(2):289294.

2. Heufelder J, Cordini D, Fuchs H, et al. Fuenf Jahre Protonentherapie von Augentumoren am Hahn-Meitner-Institut Berlin. Z Med Phys 2004;14(1):64-71.

3. Thilmann C, Nill S, Tücking T, et al. Correction of patient positioning errors based on in-line cone beam CTs: Clinical implementation and first experiences. Int J Radiation Oncology Biol Phys 2005;63(1):550-551.

4. Pluim J, Maintz J, Viergever M. Mutual information based registration of medical images: A survey. IEEE Trans Med Imaging 2003;22(8):986-1004.

5. Harris C, Stephens M. A combined corner and edge detector. In: Procs Alvey Vision Conference; 1988. 147-152.

6. Press WH, Teukolsky SA, Vetterling WT, Flannery BP. Numerical Recipes in C. vol. 2. Cambridge University Press; 1992.

7. Huttenlocher DP, Klanderman GA, Rucklidge WJ. Comparing images using the Hausdorff distance. IEEE Trans PAMI 1993;15(9):850-863. 